\title{
The Role of Wine Polysaccharides on Salivary Protein-Tannin Interaction: A Molecular Approach
}

\section{Elsa Brandão}




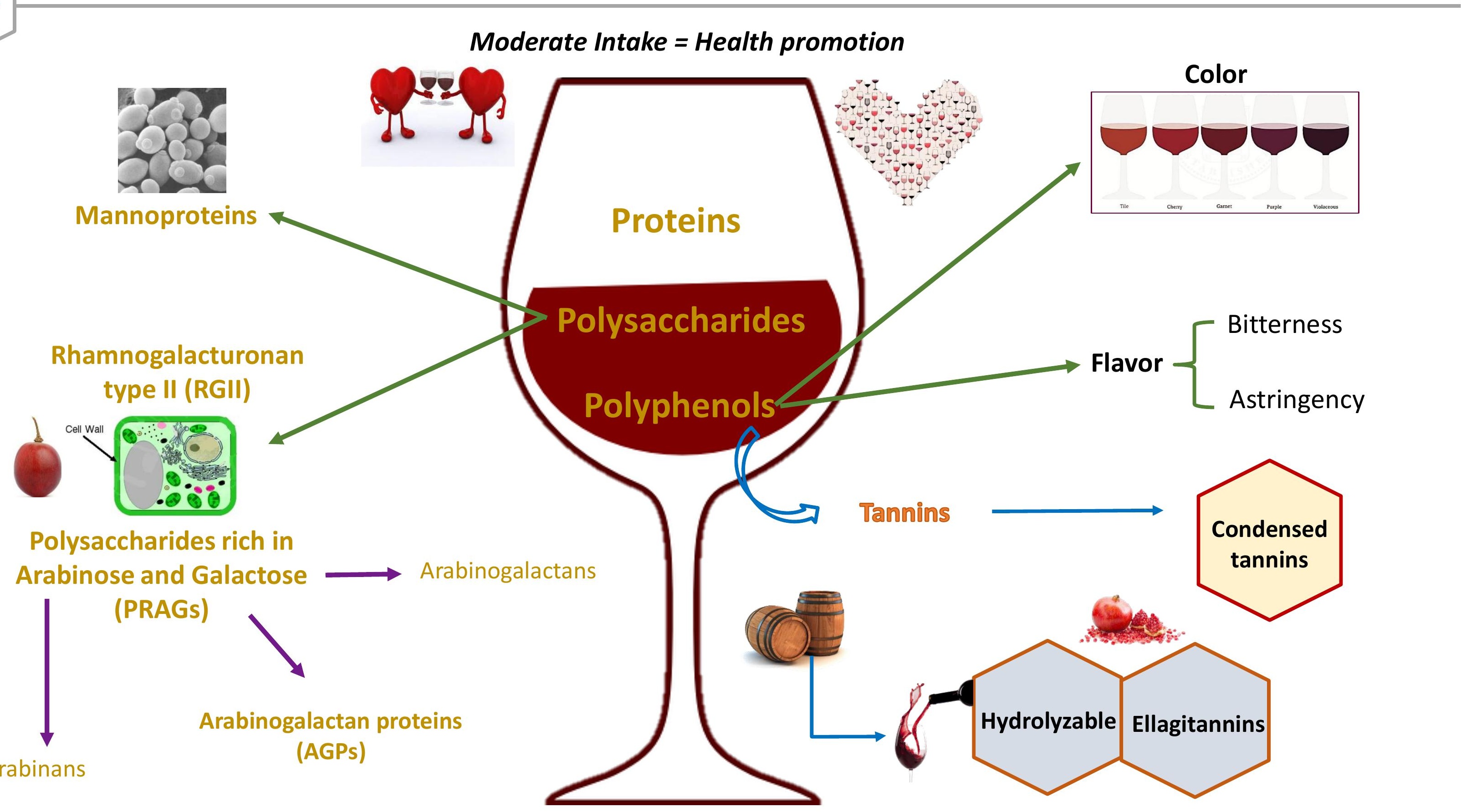




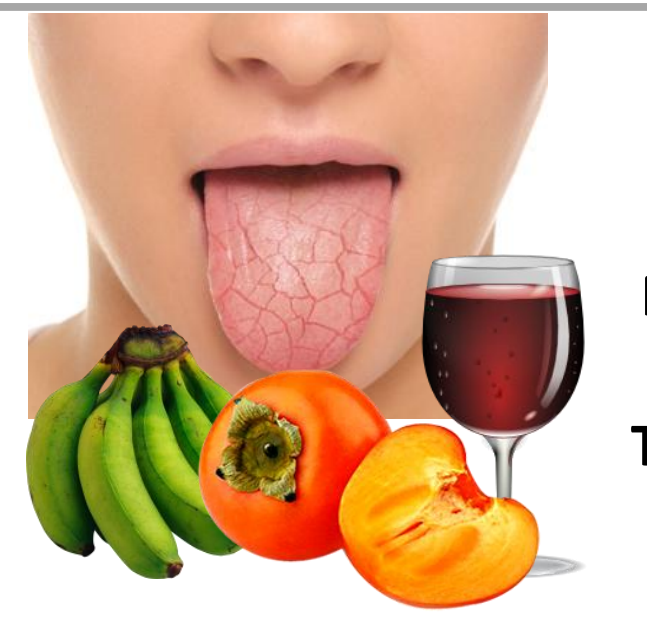

\section{Dryness}

\section{Puckering}

Tightening
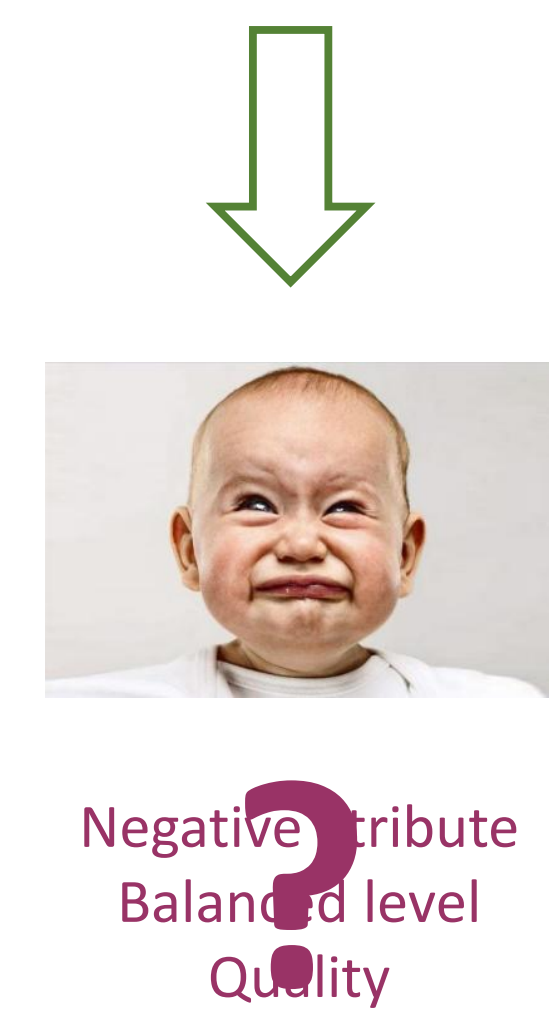

Negative tribute Baland level Quelity

\section{Mechanisms of Astringency}

- Mechanoreceptors

- Interaction with oral cells

- Salivary proteins/Tannins interaction

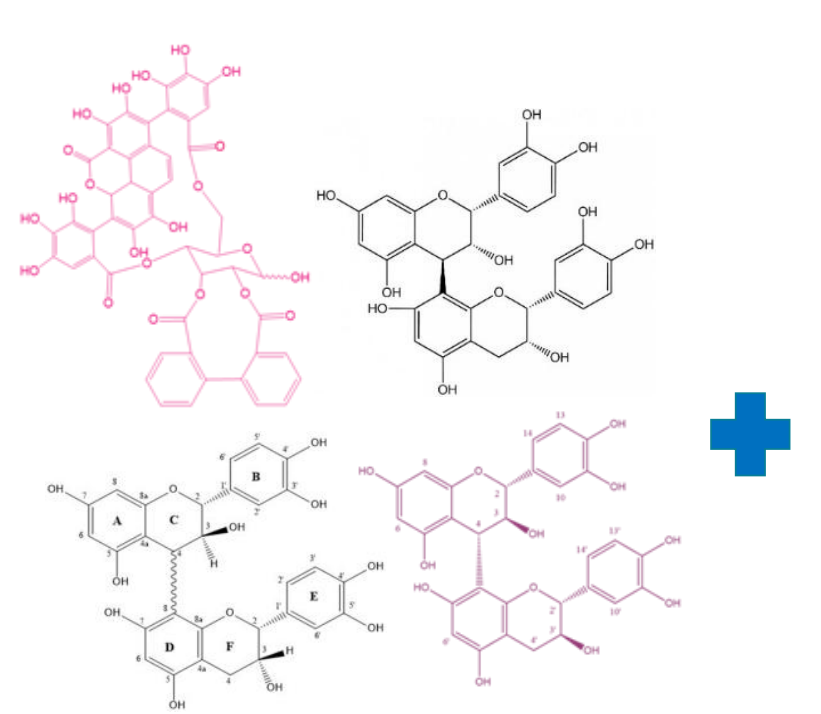

Tannins

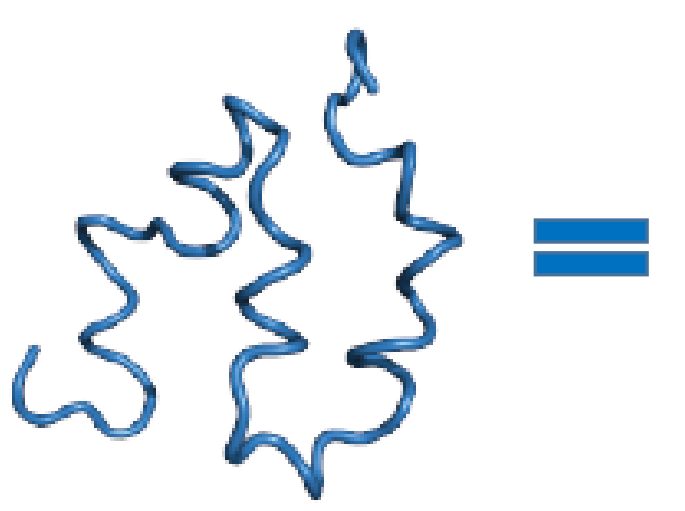

Salivary Proteins

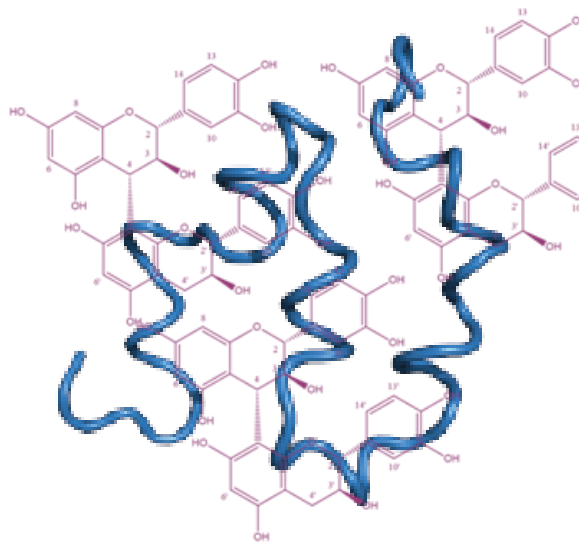

(In)Soluble Complexes 

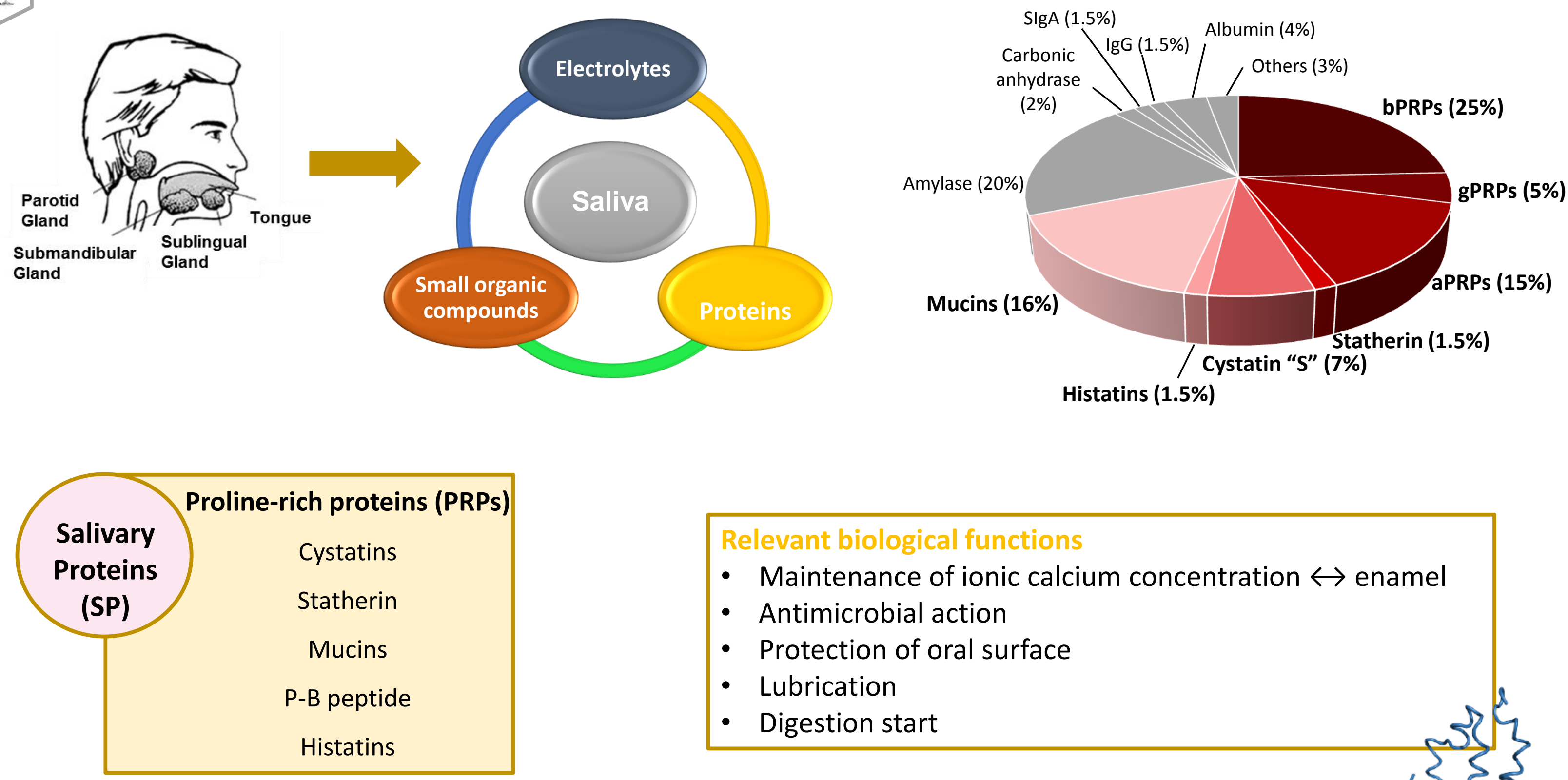

Histatins (1.5\%)

Relevant biological functions

- Maintenance of ionic calcium concentration $\leftrightarrow$ enamel

- Antimicrobial action

- Protection of oral surface

- Lubrication

- Digestion start 


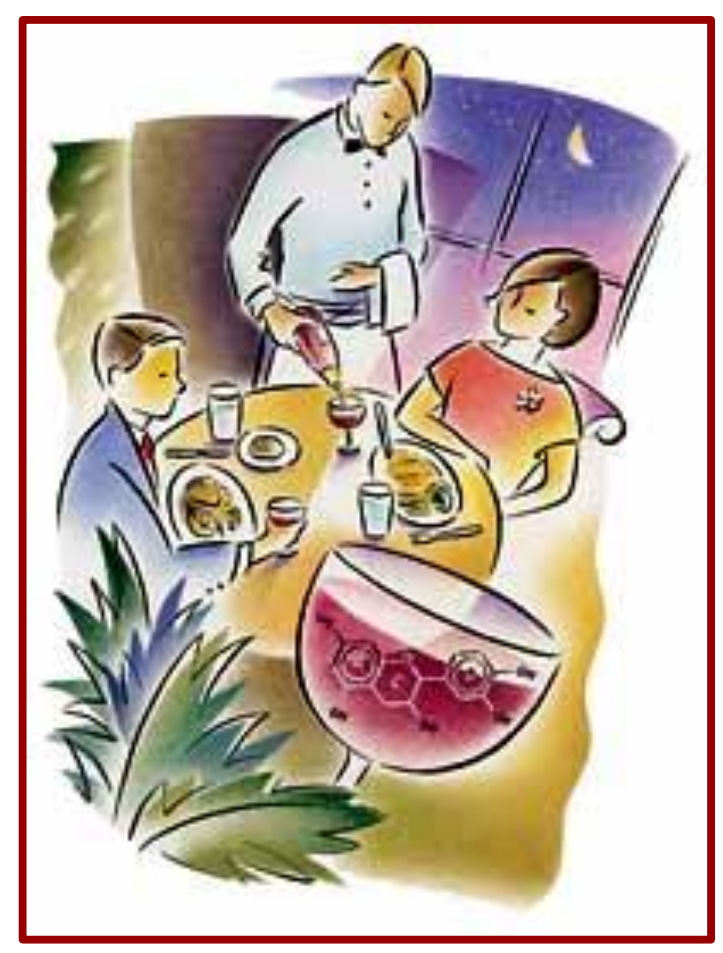

\section{Astringency Modulation}
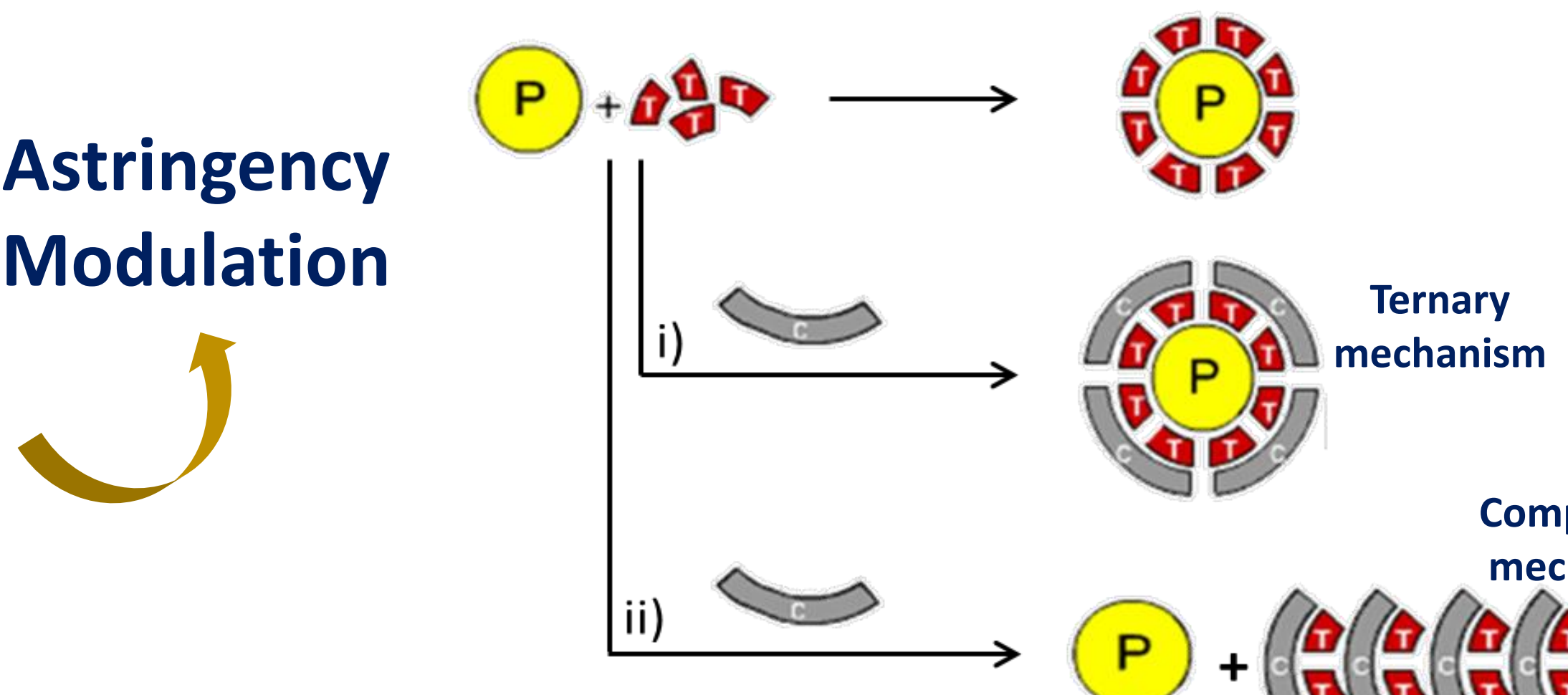

ii)

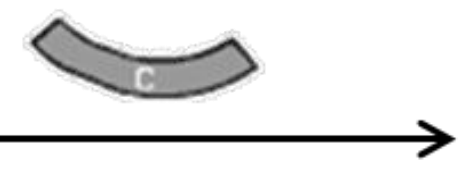

Competition mechanism

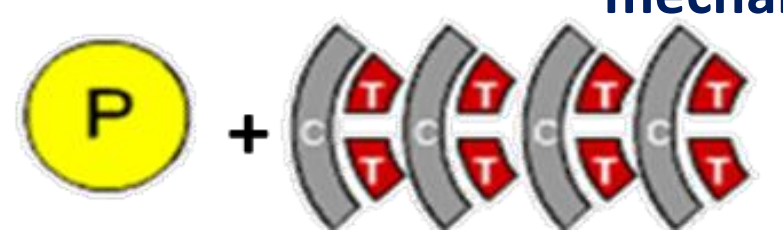

Ii) Polysachharides could form ternary complexes with the protein-tannin aggregates, enhancing its solubility in
ii) Polysaccharides have the ability to inhibit protein-tannin interaction by competing with SP to bind tannins. 

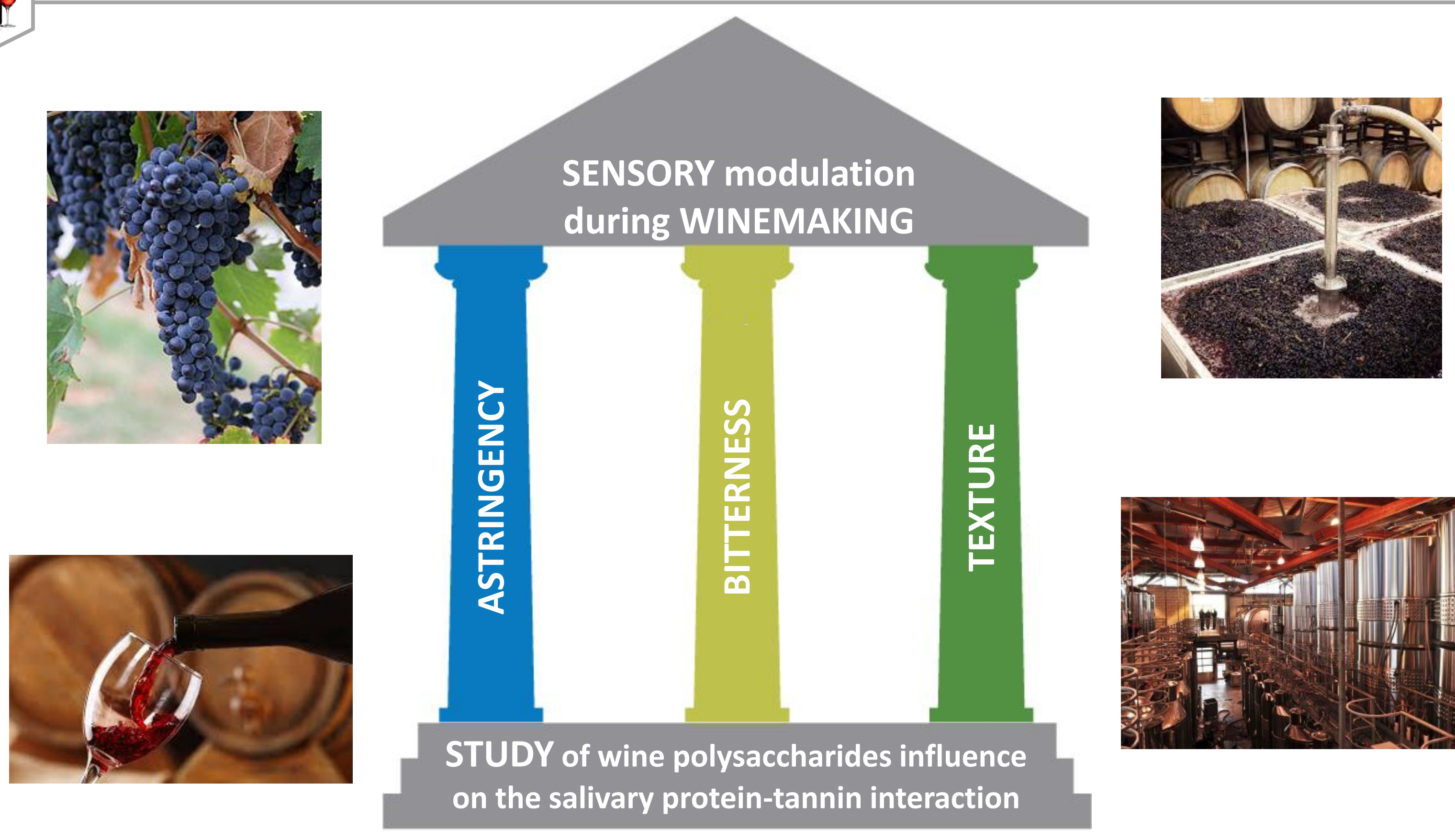
on the salivary protein-tannin interaction

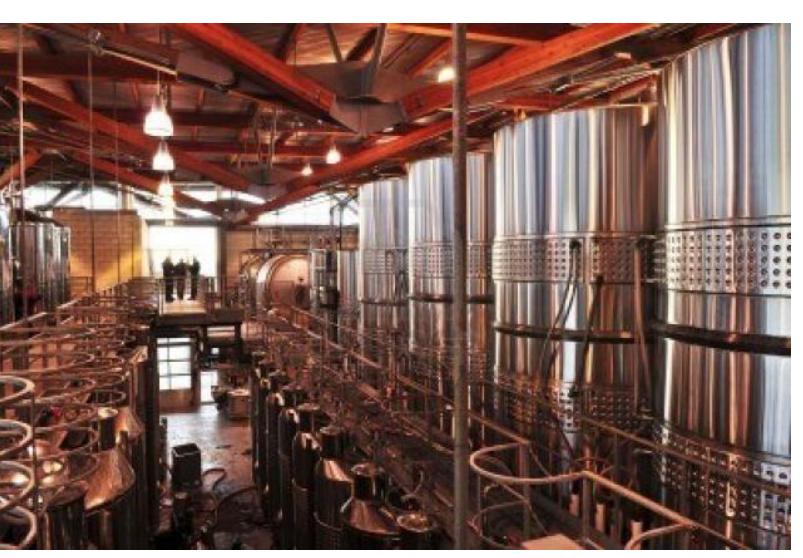




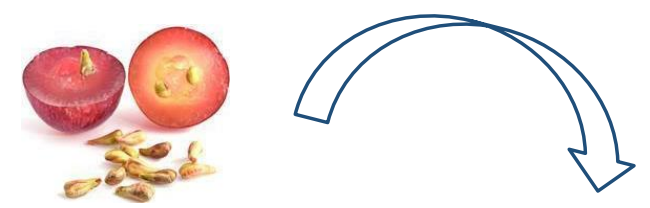

Procyanidin B2
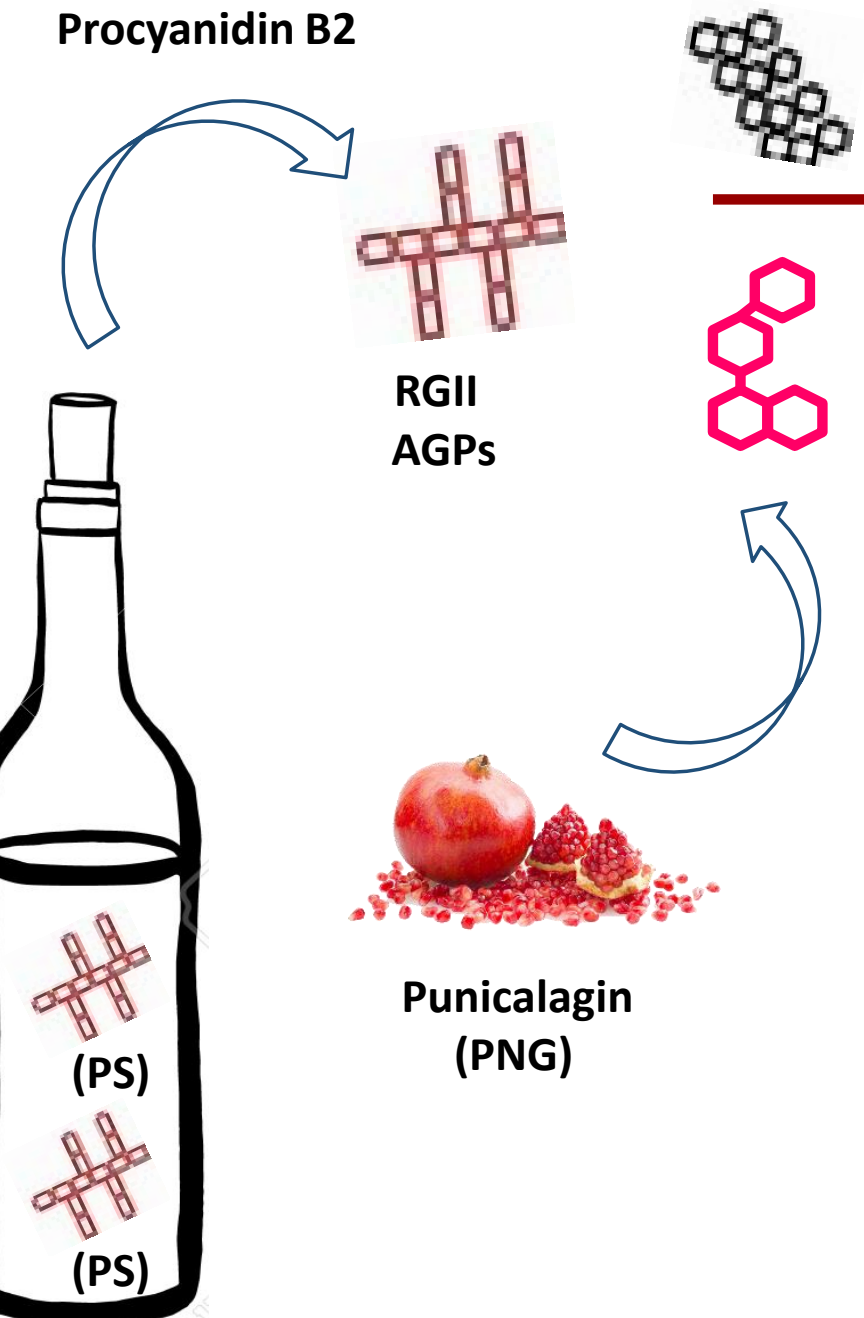

RGII

AGPs

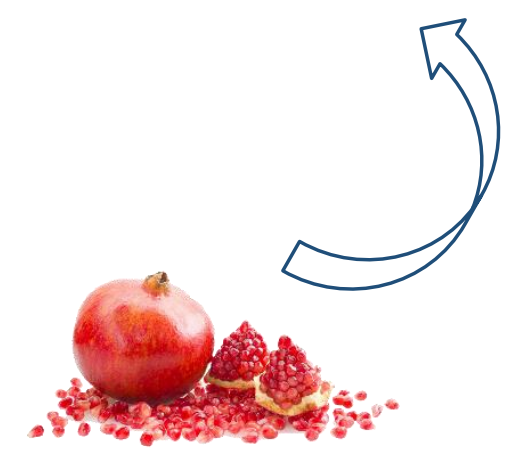

Punicalagin

(PNG)
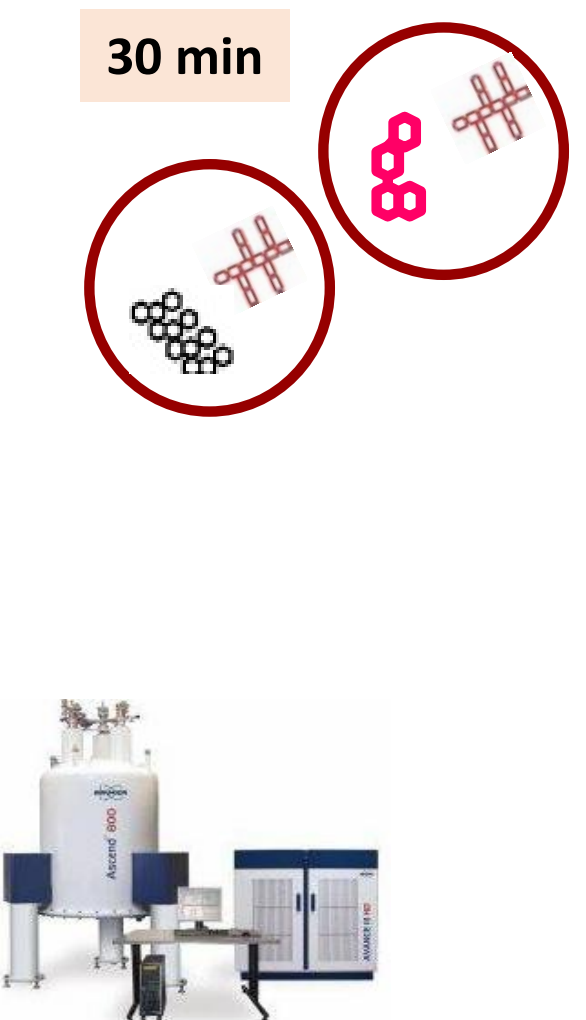

Saturation-Transfer Difference (STD)-NMR

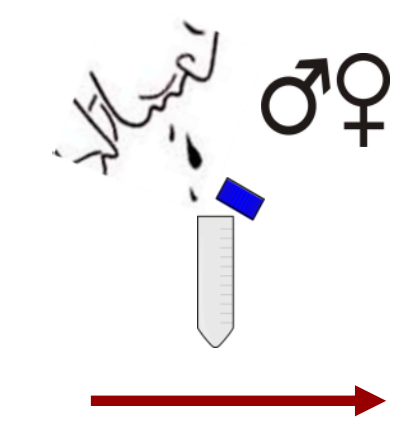

Acidic PRPs (aPRPs) P-B peptide

HPLC/Nephelometry
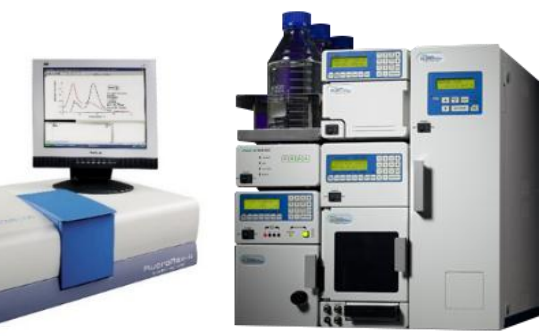

Precipitation/Aggregation studies

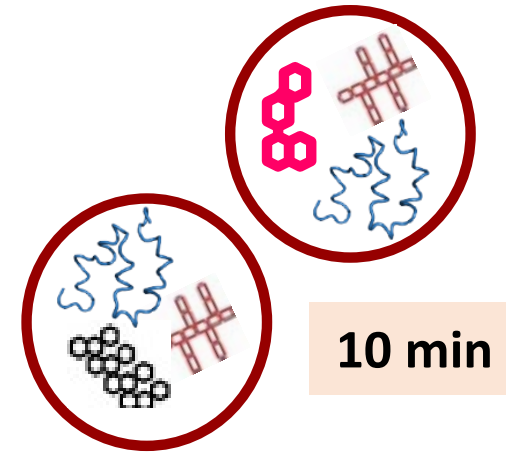

10500 rpm, $5 \mathrm{~min}$

Polysaccharides (PS) 


\section{Isolation and characterization of wine polysaccharides}
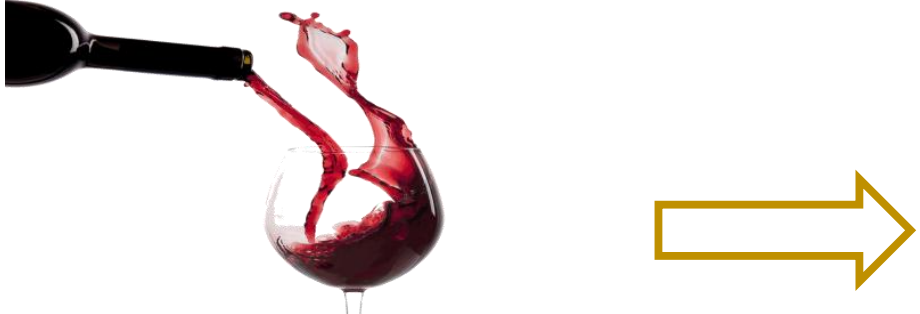

- Adsorption chromatography

- Anion-exchange chromatography

- Size exclusion high resolution column chromatography

- Affinity chromatography
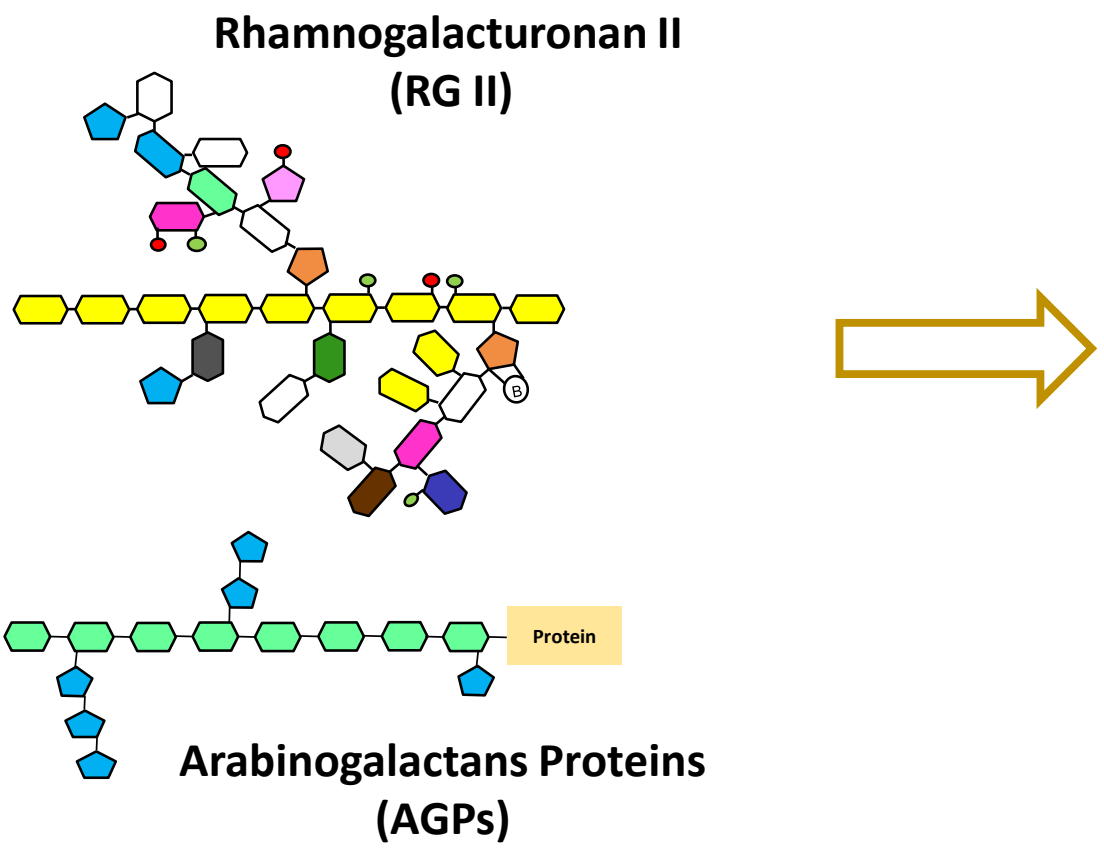

GC-MS analysis of the individual glycosyl residues

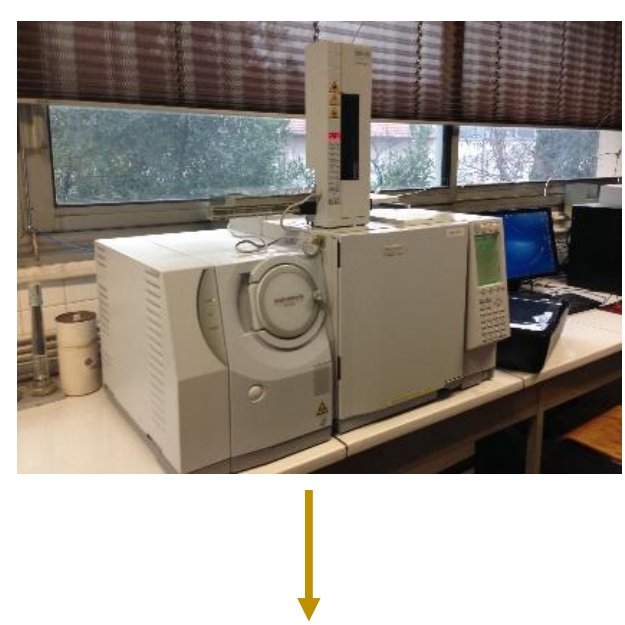

Neutral and acidic sugar determination

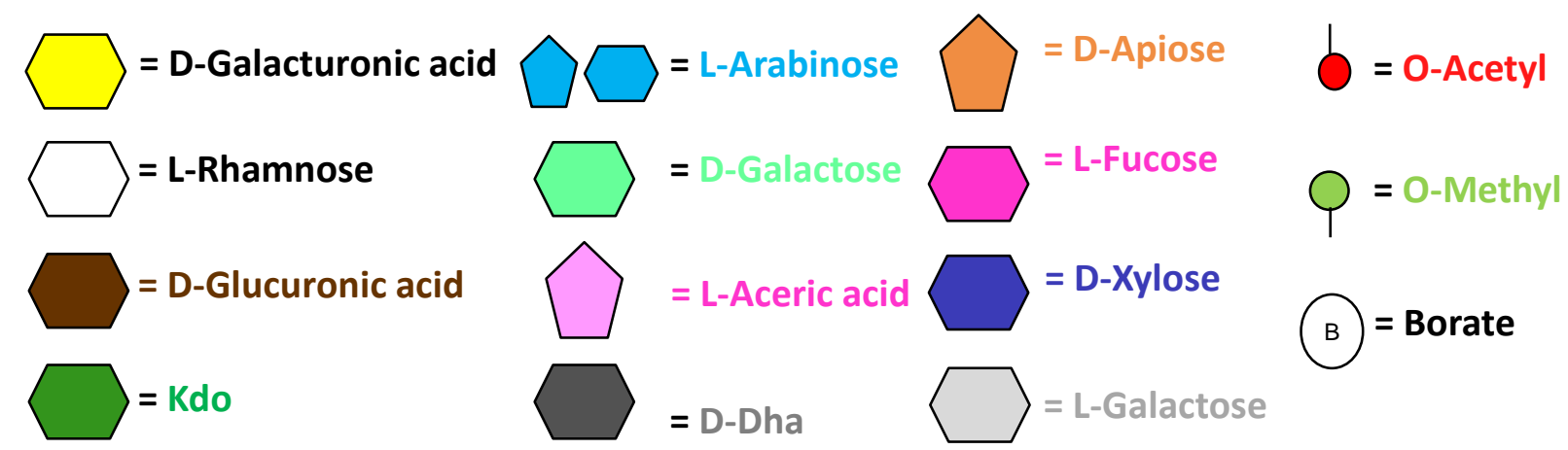




\section{Nephelometry}

$90^{\circ}$ light scattering photometer $\lambda$ exc $=\lambda$ em $=400 \mathrm{~nm}$

Decrease in light scattering

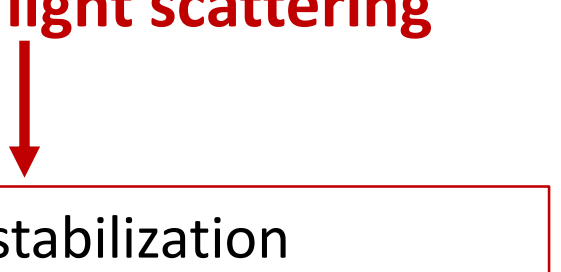

\section{Influence of polysaccharides on SP-Tannin aggregation}
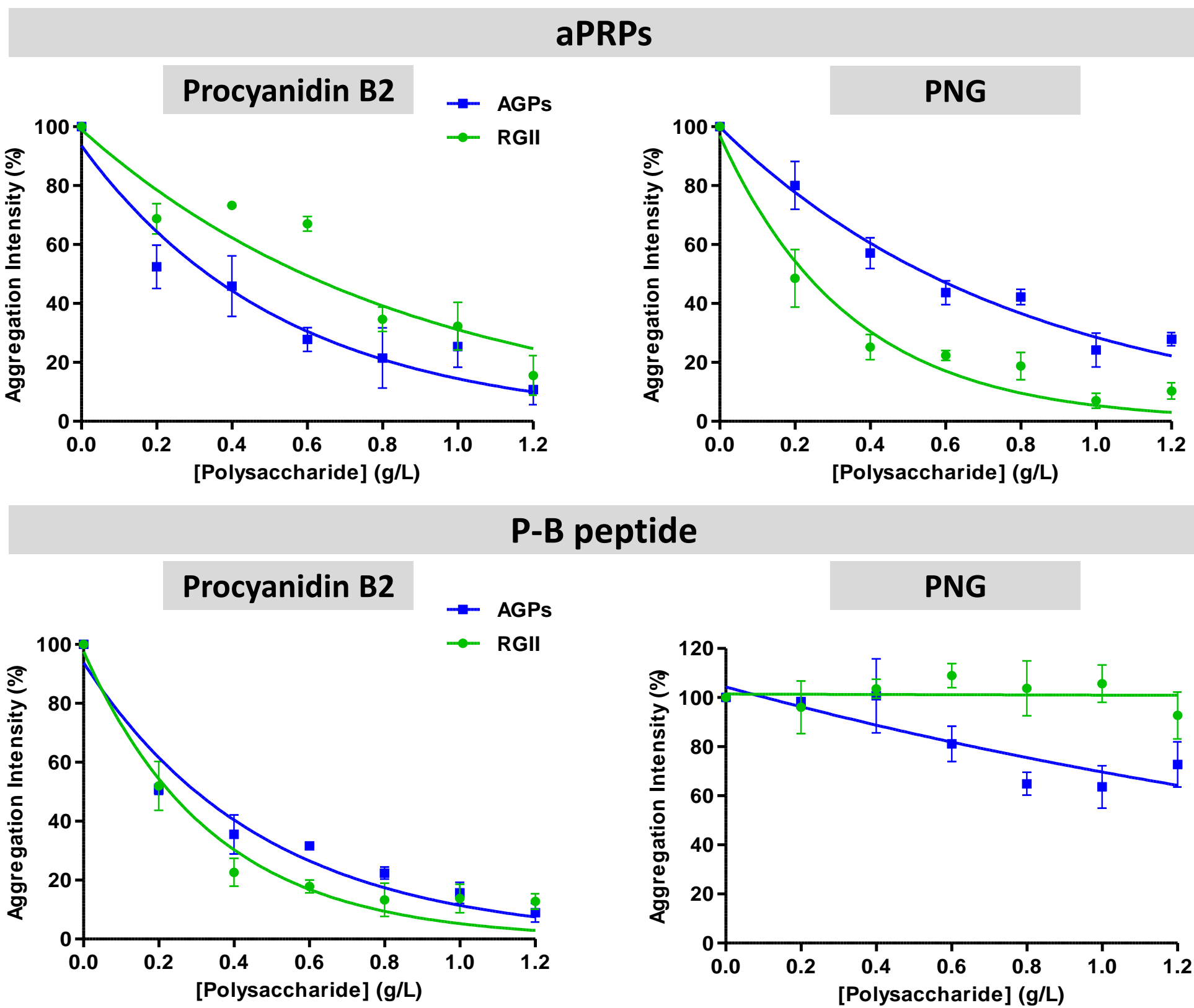

PNG

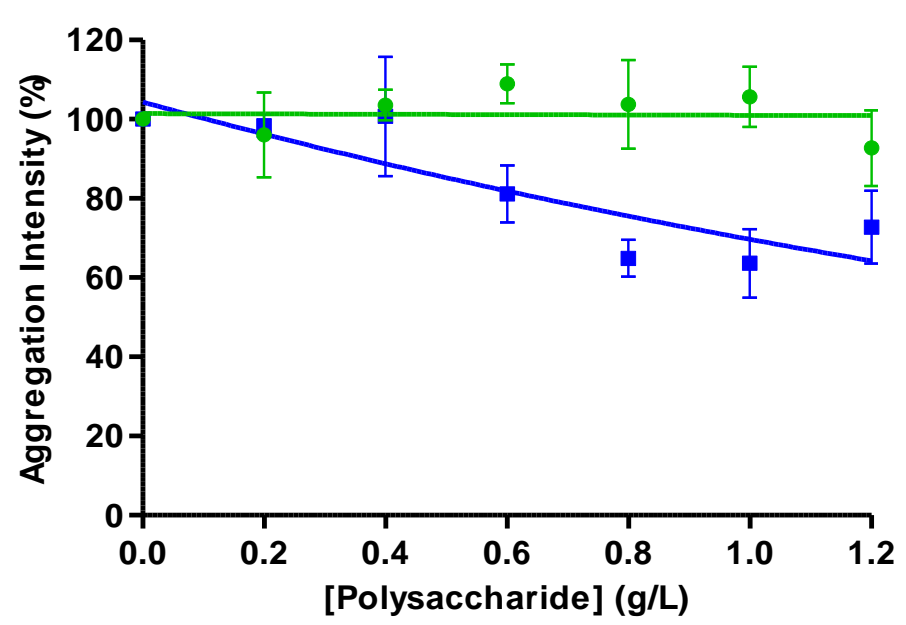




\section{STD-NMR results}

B2

\section{PNG}

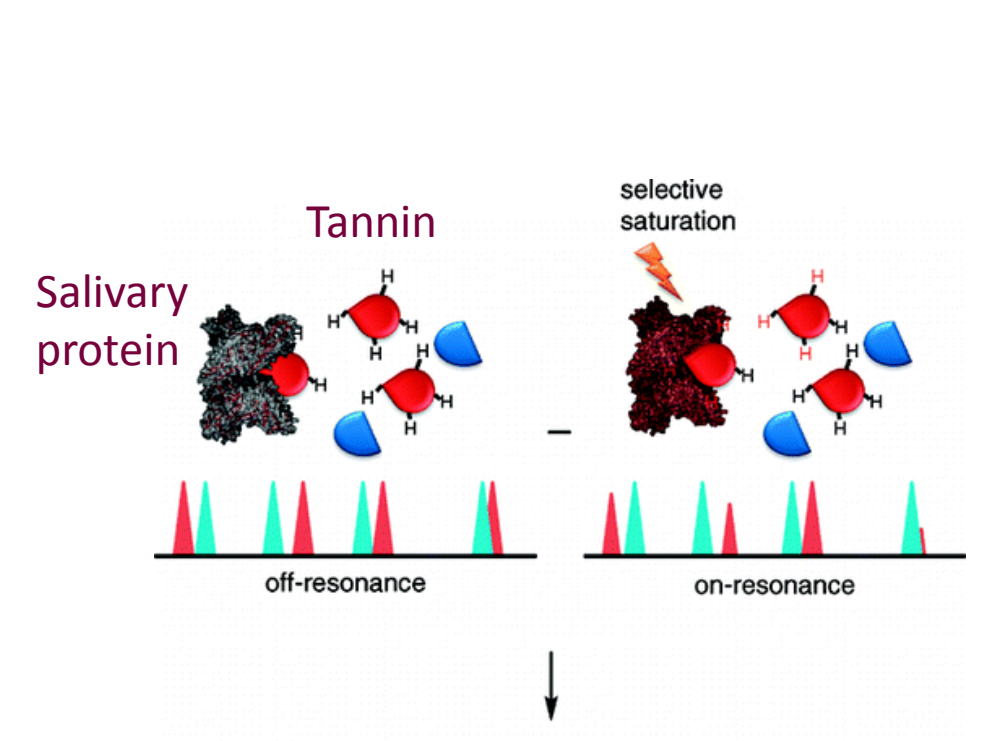

STD - difference spectrum

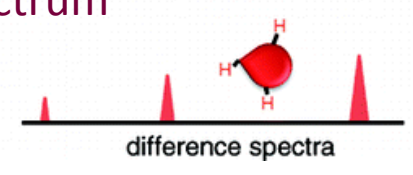

STD tannin signal

\begin{tabular}{c|ccc|c|}
\hline & AGPS & RGII & AGPS & RGII \\
\cline { 2 - 4 } aPRPs & 65.4 & 42.9 & 57.7 & 89.1 \\
\hline P-B peptide & 83.0 & 87.6 & 86.3 & 99.8 \\
\hline
\end{tabular}

\section{STD tannin signal \\ Polysaccharides bind tannins, competing with SP \\ Competition mechanism}

Formation of a ternary complex SP-Tannin-Polysaccharide

Ternary mechanism

aPRPs + Procyanidin B2 + AGPs $0.8 \mathrm{~g} / \mathrm{L}$ aPRPs + Procyanidin B2 (Control)
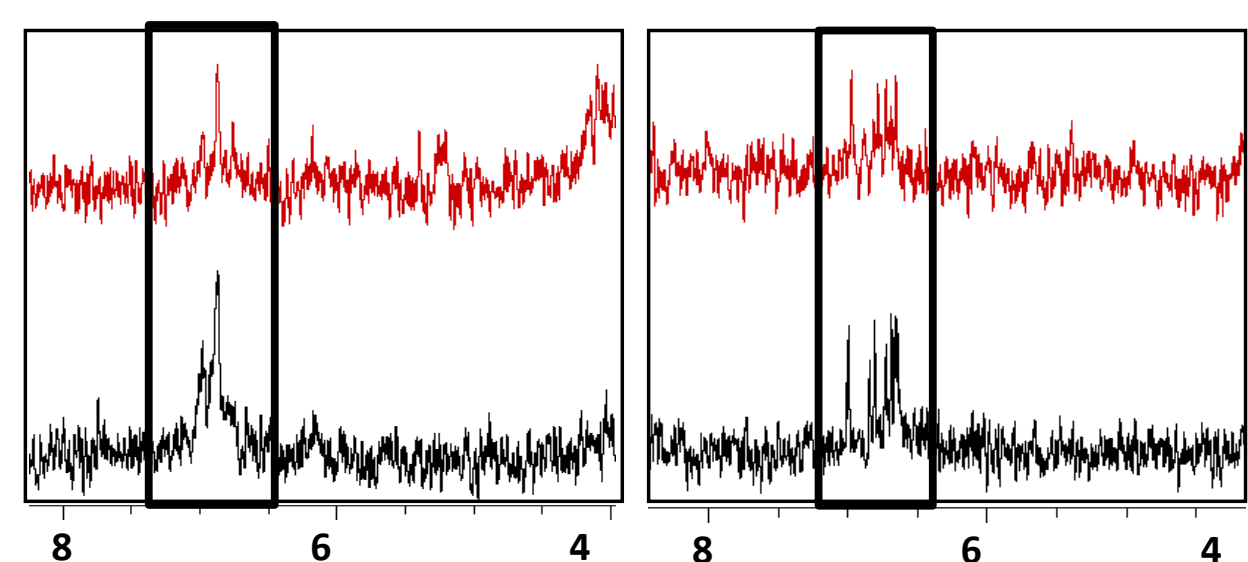

aPRPs + PNG + AGPs $0.8 \mathrm{~g} / \mathrm{L}$

aPRPs + PNG

(Control) 
Wine polysaccharides are able to reduce or inhibit the interaction between SP and tannins

HPLC and nephelometry results showed that there is non-association (competition mechamism) or (re)solubilization of SP-tannin aggregates (ternary mechanism)

STD-NMR results - suggest which mechanism was responsibe for each interaction

B2

AGPs

Competition

Ternary
RGII

Competition Ternary

PNG

AGPs

Competition

Ternary

Differences of polarity of the molecules involved

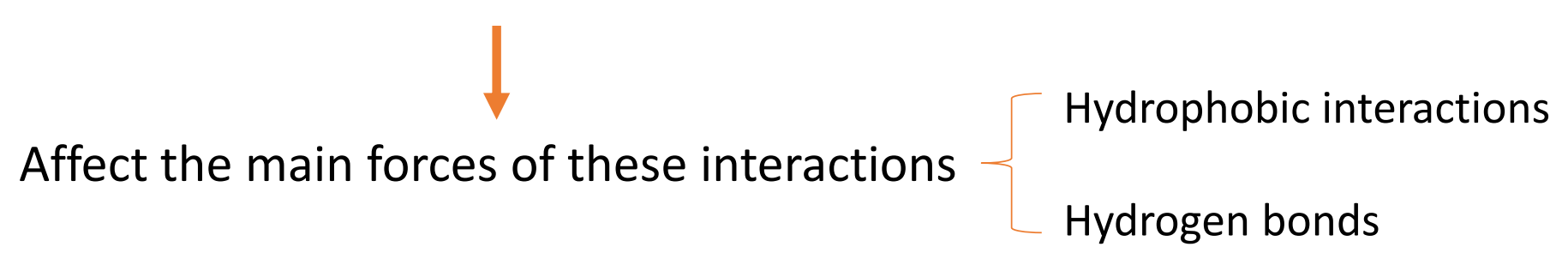

Hydrophobic interactions

Hydrogen bonds

RGII

Ternary

\section{P-B peptide}

$\checkmark$

$-$




\section{THANK YOU FOR YOUR ATTENTION!}

\section{Acknowledgements}

\section{U.PORTO}

Fu faculdade de ciências

$C \begin{aligned} & \text { FACULDADE DE CIÉNCIAS } \\ & \text { UNIVERSIDADE DO PORTO }\end{aligned}$

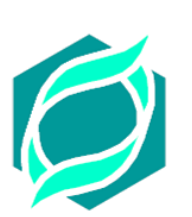

i c e t a

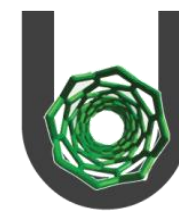

LAQV

@ REQUIMTE

(b) Interreg

España - Portugal

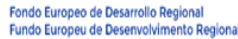

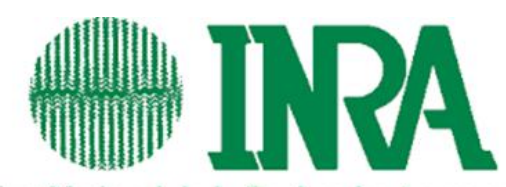

Institut National de la Recherche Agronomique CENTRE DE MONTPELLIER

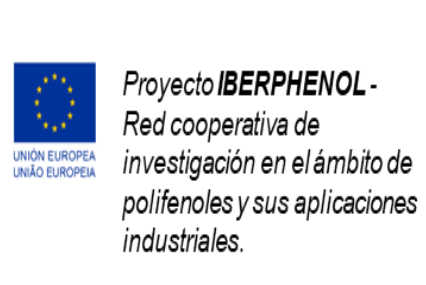

\section{C@MPETE 2020

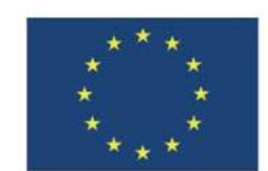 \\ UNIĀO EUROPEIA \\ Fundo Europeu \\ de Desenvolvimento Regional}
Victor de Freitas
Susana Soares
Nuno Mateus
Ignacio García-Estévez
Thierry Doco
Mafalda Santos Silva

Pascale Williams

\section{Financial support}

PTDC/AGR421-TEC/6547/2014

UID/QUI/50006/2013-POCI/01/0145/FEDER/007265

SFRH/BPD/88866/2012

SFRH/BD/105295/2014 


\section{www.foodphenolab.com}

Joana Oliveira Joana Azevedo Iva Fernandes Ana Fernandes Ana Évora Ricardo Dias Rosa Perez-Gregorio Luis Cruz Hélder Oliveira Paula Araujo Natercia Teixeira Marta Guimaraes Vania Gomes Telmo Francisco Abigail Ferreira Sofia Reis Ana Reis Erika Salas Raul Ferrer Joana Brás
Victor de Freitas Nuno Mateus Elsa Brandão Susana Soares Mafalda Silva Ignacio García-Estévez
U.PORTO

FCu vaculdade de ciências

FCT

Fundação para a Ciência e a Tecnologia @ REQUIMTE
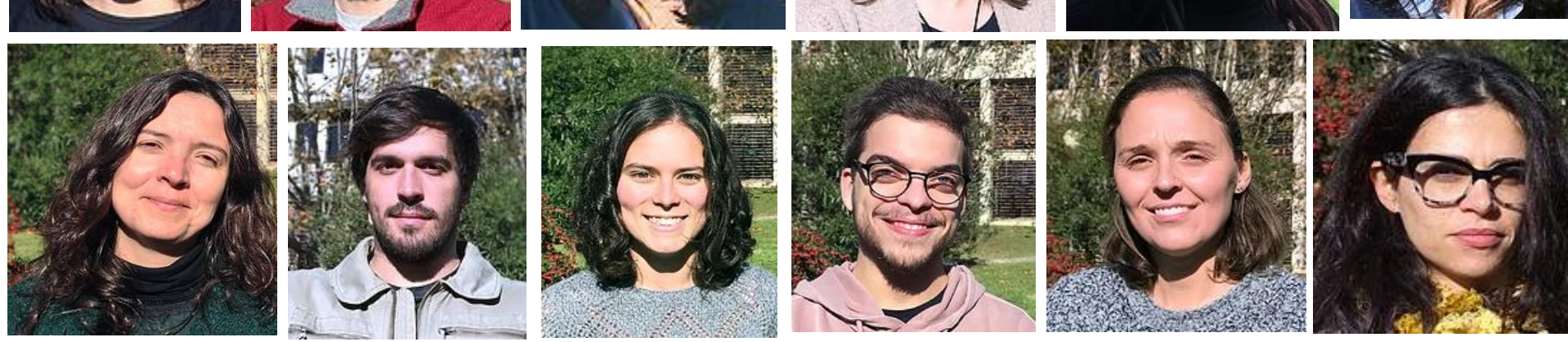
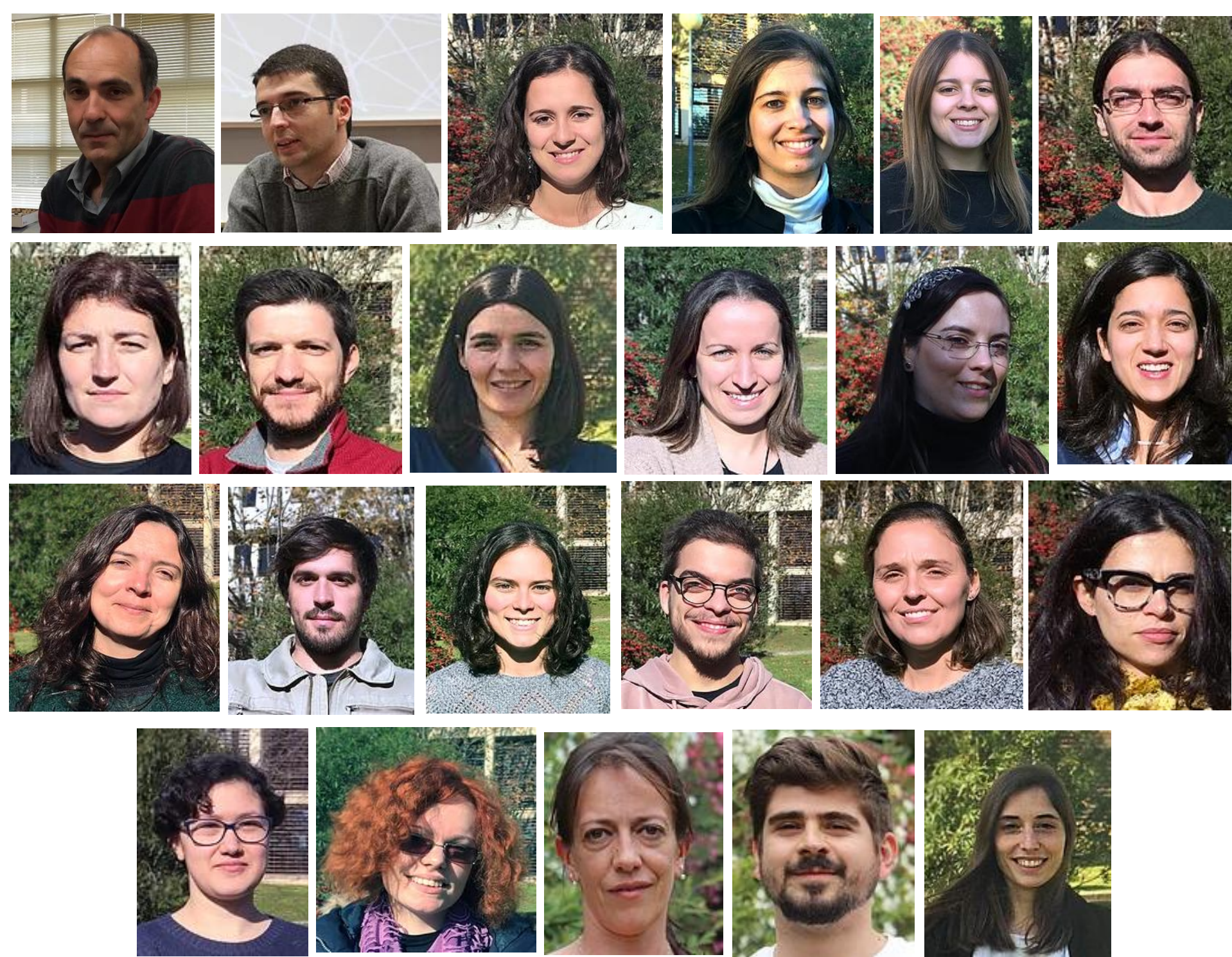